\title{
THA First: Low Back Pain in Patients with Both Hip Osteoarthritis and Lumbar Degenerative Disease is Relieved after Total Hip Arthroplasty
}

\section{Tianfei Ran}

Xinqiao Hospital

Song Ke

Xinqiao Hospital

Mingrui Lv

Xinqiao Hospital

Jie Li

Xinqiao Hospital

\section{Xin Song}

Xinqiao Hospital

\section{Rui Zhang}

Army Medical University

Yuanyuan Zhou

Xinqiao Hospital

Min Wang ( $\nabla$ xqwangmin@sina.com )

Xinqiao Hospital

\section{Research article}

Keywords: arthroplasty (THA), postoperatively, postoperatively

Posted Date: September 10th, 2020

DOI: https://doi.org/10.21203/rs.3.rs-64741/v1

License: (1) (1) This work is licensed under a Creative Commons Attribution 4.0 International License. Read Full License 


\section{Abstract}

Background: With the aging of population, the number of patients suffering from hip osteoarthritis complicated with lumbar degenerative disease is increasing. Both diseases may require surgery, however, which is first? the sequence of total hip arthroplasty (THA) and lumbar surgery remains controversial.

Methods: The data of our hospital, from 2010 to 2019 were retrieved, and a group of patients who had undergone total hip replacement (THA, $n=153$ ) and also suffered from lumbar degenerative diseases were identified. We studied the improvement of hip joint function and the relief of low back pain (LBP), and also discussed the effect of unilateral and bilateral THA on the relief of LBP.

Results: The LBP visual analysis scale (VAS) of 153 patients decreased from (4.13 \pm 1.37$)$ preoperatively to $(1.90 \pm 1.44)$ postoperatively. The average Harris Hip score (HHS) increased from $45.33 \pm 13.23$ preoperatively to $86.44 \pm 7.59$ postoperatively of the latest follow-up. According to Japanese Orthopedic Association (JOA) scoring system, the proportion of patients with good response to treatment in the clinical results of these 153 patients reached $93.46 \%$. LBP VAS decreased from $4.18 \pm 1.38$ preoperatively to $1.95 \pm 1.49$ postoperatively in unilateral group and from $3.94 \pm 1.32$ preoperatively to $1.73 \pm 1.23$ postoperatively in bilateral group respectively. These results showed that the lumbar pain was relieved significantly after surgery.

Conclusion: THA can relieve LBP while relieve hip pain and restore hip function. And the relief of LBP and recovery of lumbar function in unilateral operation group were better than those in bilateral operation group. These findings can be helpful to surgeons in making decisions. For patients with both hip disease and lumbar degenerative disease, THA first may bring additional benefits to patients.

\section{Background}

According to statistics, one in two patients with symptomatic hip joint degeneration suffers from low back pain (LBP) ${ }^{[1]}$. Along with the social aging intensifies, more and more patients have back-pain and lower-limb discomfort caused by hip joint disease and lumbar degenerative disease ${ }^{[2-6]}$. It is reported that among patients undergoing total hip arthroplasty (THA), the proportion of patients suffering from different degrees of low back pain ranged from $21.2 \%{ }^{[7,8]}$ to $60.4 \%^{[9,10]}$. Some of the symptoms of these two diseases (hip joint disease and lumbar degenerative disease) are the same, such as lower limb pain, pain around the knee joint and may have abnormal gait and LBP [11-13]. Therefore, it is difficult to identify which disease is causing the patient's current symptoms, and even more difficult to diagnose when both diseases occur in the same patient. At the same time, in the treatment of those patients, the problem on which surgery (hip surgery or lumbar surgery) is the first will occur. Up to now, the operation sequence has been controversial ${ }^{[3,5,6]}$. If a proper surgical sequence is found and accepted by both joint and spinal surgeons, preoperative counseling will be easier, patients will get better treatment, and even the associated treatment burden will be reduced. 
So far, there are few studies on the function improvement and pain relief of hip joint and LBP relief after THA in patients with the above two kinds of diseases, and there is no study yet to analyze the effect of unilateral and bilateral THA on the relief of LBP. Therefore, the purpose of this study is to follow up a group of patients with hip joint disease and lumbar degenerative disease, observe their hip joint pain relief and function recovery as well as LBP relief after THA, and explore the effect of unilateral THA and bilateral THA on such patients, so as to provide some advice for surgeons in the treatment of such patients.

\section{Methods}

\section{Patients}

All subjects received and accepted informed consent before participating in the study, which was approved by the institutional review committee of our hospital. The subjects were patients from the orthopedic department of our hospital from 2010 to 2019 who finally received THA treatment because of hip joint degenerative disease.

\section{Methods}

We searched the hospital database for patients who underwent unilateral or bilateral THA in orthopedic department from 2010 to 2019 (Before 2010 the patient information could not be obtained because the patient database was not ready.) Inclusion criteria were patients who had been diagnosed with hip arthropathy and also been diagnosed with lumbar degenerative disease but only received THA. Hip joint lesions include osteoarthritis, necrosis of the femoral head or both. Lumbar degenerative diseases include lumbar disc herniation (LDH), degenerative lumbar spondylolisthesis (DLS), degenerative scoliosis (DS) and lumbar spinal stenosis (LSS).(Fig. 1) Hip joint diseases and lumbar degenerative diseases were diagnosed by experienced joint surgeons through imaging examination including X-ray, computed tomography (CT), magnetic resonance imaging (MRI), combined with the current symptoms. The exclusion criteria were: patients who had undergone lumbar fusion or internal fixation surgery, or had vascular claudication, history of major trauma, diabetic polyneuropathy, lumbar and pelvic infections, tumor diseases, or who had undergone THA because of femoral neck fracture or ankylosing spondylitis. Patients who met any of the exclusion criteria must be excluded from the study.

The patients' height, weight, gender, age, phone number and operation time were obtained from the database. Harris Hip score (HHS) was routinely evaluated before THA. For patients with lumbar diseases, Japanese Orthopedic Association (JOA) score and low back pain (LBP) visual analysis scale (VAS) score were also evaluated. Such preoperative evaluation makes this study possible. We also collected the results of the latest follow-up. If the patient underwent lumbar surgery again because of the aggravation of lumbar pain or had reoperation for other reasons, only the score before reoperation was included in statistics. 
HHS was used to evaluate the recovery of hip joint function and pain relief[14]. JOA (0-29) score system was used to evaluate lumbar function and consisted of three parts, including symptoms (9 points), signs including including straight-leg raise (6 points), and seven activities of daily living (ADL, 14points)[15]. The score 29 represents normal function, and 0 represents the worst. According to JOA score system recovery rate $(\%)=$ (postoperative score - preoperative score) / (29 - preoperative score) $\times 100 \%$, RR was calculated and divided into four groups: cured ( $R R=100 \%)$, markedly effective $(60 \% \leqq R R \leqq 99 \%)$, effective ( $25 \% \leqq R R \leqq 60 \%)$ and ineffective (RR $\leqq 25 \%)$ [16]. VAS was used to evaluate the pain of the low back and the range from 0 to 10 , where 0 represents no pain and 10 the worst possible pain[17].

We analyzed the preoperative and postoperative HHS score, JOA score, and LBP VAS score of these patients. At the same time, in order to analyze the effect of unilateral and bilateral THA surgery on the relief of LBP, we divided the patients into unilateral THA surgery group and bilateral THA surgery group, and compared the difference between the two groups in follow-up results.

\section{Statistical analysis}

The data was analyzed by statistical software (with the latest version of SPSS; IBM), and the quantitative data are expressed as mean \pm standard deviation (SD). T test was used to analyze the preoperative scores and postoperative follow-up results of the 153 patients. Independent t-test was used to analyze the LBP VAS and HHS of unilateral and bilateral THA groups. A two-tailed probability level of $p<0.05$ was selected as the statistically significant level.

\section{Results}

According to the inclusion criteria and exclusion criteria, finally 160 patients met the criteria, among which 7 patients died and lost in the follow-up, and 153 patients were included into study. All patients received unilateral or bilateral THA treatment in orthopedic department of our hospital from 2010 to 2019, including 120 cases of unilateral THA and 33 cases of bilateral THA. These operations were performed by 3 experienced joint surgeons. The average age of male was $62.93(47-80)$ and that of female was $65.23(43-88)$ at the time of operation. The average follow-up time was 44.3 months (24-108 months). All patients recovered smoothly without complications. Demographic data and surgical information are shown in Table 1. 
Table 1

Demographic and Clinical Parameters of Patients

\section{Demographic and Clinical Parameter}

\begin{tabular}{|lll|}
\hline Demographic & male & female \\
\hline Gender & $69(45.1 \%)$ & $84(54.9 \%)$ \\
\hline Age in years, (mean, range) & $62.93(47-80)$ & $65.23(43-88)$ \\
\hline BMI(Kg/m2) & $24.06(17.80-33.17)$ & $24.29(15.39-33.74)$ \\
\hline Clinical Parameter & & \\
\hline Degenerative spinal disorders, $n$ & $115(75.16 \%)$ & \\
\hline LDH & $12(7.84 \%)$ & \\
\hline DLS & $6(3.92 \%)$ & \\
\hline DS & $19(12.42 \%)$ & unilateral(120) \\
\hline LSS & 6 & General(49) \\
\hline Lumber surgery after THA & bilateral(33) & \\
\hline Operative side & lumbar anesthesia (104) & \\
\hline Anesthesia type & & \\
\hline $\begin{array}{l}\text { LDH = lumbar disc herniation; DLS = degenerative lumbar spondylolisthesis; DS = degenerative } \\
\text { scoliosis; LSS = lumbar spinal stenosis, BMI = body mass index }\end{array}$ & \\
\hline
\end{tabular}

153 patients had significant relief of low back pain after surgery. The mean LBP VAS score decreased from $(4.13 \pm 1.37)$ preoperatively to $(1.90 \pm 1.44)$ postoperatively $(P<0.0001)$. According to the calculation method of recovery rate in JOA scoring system that 8 patients were cure, 66 were apparent, 61 were effective, 10 were invalid. The effective rate was $93.46 \%$. The average HHS increased from $45.33 \pm$ 13.23 preoperatively to $86.44 \pm 7.59$ postoperatively $(P<0.0001)$ (Fig. 2).

In our study, 120 patients received unilateral THA and 33 patients received bilateral THA. The follow-up results of the two groups showed significant improvement: hip joint function was improved and pain was relieved significantly, LBP was distinctly reduced. There was no significant difference in preoperative HHS and LBP VAS between unilateral and bilateral groups $(P>0.05)$. However, interestingly, the patients who received unilateral THA had better relief of low back pain and recovery of lumbar function than those who received bilateral THA, and the difference was statistically significant. (Fig. 3, Table 2) 
Table 2

Harris Hip and VAS scores of patients underwent unilateral and bilateral THA

\begin{tabular}{|lllll|}
\hline & & Bilateral $(\mathbf{n}=\mathbf{3 3})$ & Unilateral $(\mathbf{n}=\mathbf{1 2 0})$ & $\mathbf{p}$ \\
\hline Harris Hip score & Preoperative & $44.39 \pm 12.02$ & $45.59 \pm 13.59$ & 0.67 \\
\cline { 2 - 5 } & Postoperative & $87.58 \pm 5.42$ & $86.13 \pm 8.07$ & 0.33 \\
\hline LBP VAS score & $\mathrm{p}$ & $<0.000$ & $<0.000$ & 0.37 \\
& Preoperative & $3.94 \pm 1.32$ & $4.18 \pm 1.38$ & 0.43 \\
\cline { 2 - 4 } & Postoperative & $1.73 \pm 1.23$ & $1.95 \pm 1.49$ & \\
\hline Bold denotes statistically significant at $\mathrm{P}<0.05$. All values are statistically calculated as Mean \pm SD. \\
\hline
\end{tabular}

Although most of the patients' pain relieved or even disappeared, there were 9 patients with persistent or aggravated pain after operation. Among them, 6 patients underwent lumbar surgery (The pain was relieved in 5 patients after reoperation and not in 1 patient) and other 3 patients chose conservative treatment for pain. Their information are shown in Table 3.

Table 3

Reoperation data of 6 patients after THA.

\begin{tabular}{|c|c|c|c|c|c|}
\hline Case & diagnosis & Hip surgery & Spine surgery & Interval(years) & Pain \\
\hline 1 & Hip OA + LDH & \multirow[t]{6}{*}{ unilateral THA } & MIS-TLIF & 1 & relief \\
\hline 2 & Hip OA + LDH & & MIS-TLIF & 1.5 & relief \\
\hline 3 & Hip OA + LDH & & MIS-TLIF & 1.8 & relief \\
\hline 4 & Hip OA + LDH & & MIS-TLIF & 2.5 & persistent \\
\hline 5 & Hip OA + DLS & & LSF & 2 & relief \\
\hline 6 & Hip OA + LSS & & PVP & 1 & relief \\
\hline \multicolumn{6}{|c|}{ 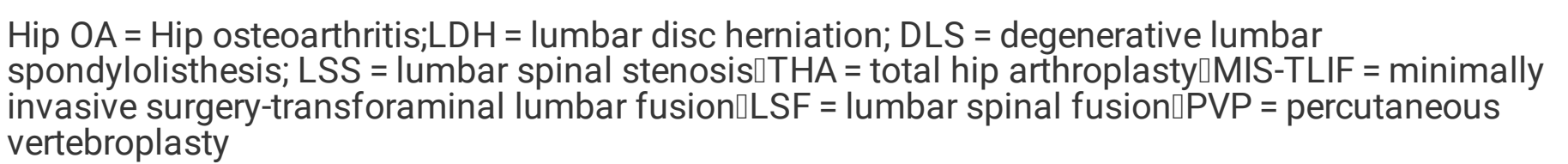 } \\
\hline
\end{tabular}

\section{Discussion}

In this retrospective study, we followed up 153 patients who were diagnosed with both hip joint disease and lumbar degenerative disease before surgery. They all received THA. These operations were performed by 3 experienced joint surgeons. Through our follow-up, it was found that the symptoms of LBP in these patients eased with the relief of hip pain and improvement of hip function after THA. Their average LBP 
VAS score decreased from preoperative $(4.13 \pm 1.37)$ to postoperative $(1.90 \pm 1.44),(p<0.0001)$. At the same time, according to the RR calculation formula of the JOA scoring system, the proportion of patients with good treatment response reached $93.46 \%$. Our results show that LBP has been relieved after THA surgery, which is consistent with previous studies ${ }^{[9,10,18]}$. Although most patients' pain was relieved or even disappeared, there were 9 cases of persistent or worsening pain postoperatively. 8 of them achieved good results after receiving medications or another lumbar spine surgery (Table 3 ).

To the best of our knowledge, this study is the first to discuss the effects of unilateral and bilateral THA surgery on patients with both hip and lumbar degenerative diseases. In our study, it was found that the follow-up results of patients in the unilateral and bilateral surgery groups were both significantly improved. Although there was no significant difference in preoperative and postoperative Harris and LBP VAS of hip joints between the unilateral surgery group and the bilateral surgery group, it is interesting to find that the LBP VAS of patients who received unilateral THA changed greater and the difference was statistically significant. This means that patients receiving unilateral THA treatment have better pain relief than patients receiving bilateral THA treatment. It has been reported that patients receiving bilateral THA had higher hip pain level preoperatively than that in the unilateral group, but there was no difference in the scores between the two groups after 1 year of surgery, which is somewhat not consistent with our preoperative results of HHS [19]. The difference in preoperative scores may be related to our small sample. Since our study is the first to discuss the effects of unilateral and bilateral THA treatment on these patients, it is impossible to compare the results we obtained with other researchers.

There are many possible reasons for LBP relief after hip joint surgery. We believe that the following reasons may be helpful in the interpretation of the follow-up results obtained in this study: (1) some studies have shown that THA may help improve the parameters of the spine and pelvis of the patient to restore the balance of the sagittal plane of the spine. Such changes may relieve low back pain and improve lumbar function [20-23]. (2) Changes in walking posture and daily life activities of patients after hip joint surgery can correct the poor postures leading to spinal diseases. (3) After hip surgery, hip pain is effectively relieved, and the quality of life is improved, which relieves the tension and anxiety caused by the patient's pain, thereby reducing the impact of LBP on the patient. However, the mechanism for the relief of LBP after THA is complicated, which requires more in-depth study and investigation to expound. In particular, the pain relief in the unilateral surgery group is more obvious, which may become a new breakthrough in exploring the relief of low back pain after THA.

Offierski and MacNab first described this connection between hip osteoarthritis (OA) and spine disease as hip spine syndrome (HSS) in 1983[24]. Failure to recognize this close pathological relationship between the spine and hip joint may delay treatment and lead to unsatisfactory surgical outcomes of the hip or spine $[25,26]$. Some patients with hip disease and lumbar degenerative disease may show the same or similar symptoms: hip and lower limb pain, sometimes including pain around the knee, gait abnormalities, changes in the sagittal sequence of the lumbar spine, and low back pain symptom. Many literatures reported that the sagittal imbalance of the spine is correlated with low back pain and hip symptoms, and a variety of measurement methods have been used to confirm. Therefore, the facts above 
will cause great trouble for joint surgeons to do diagnosis. What's more, in the treatment of such patients, the problem on which of hip or lumbar spine surgery is first will arise. So far, the sequence of surgery for patients with hip osteoarthritis and lumbar degenerative disease has been controversial.

Previous studies have shown that the surgical sequence may bring different effects to patients $[8,11,27$, 28]. There is no doubt that THA can effectively relieve pain and restore function in patients with advanced hip arthritis $[11,29,30]$. At the same time, some related studies have shown that patients with coexisting hip and lumbar degenerative diseases have treated lumbar diseases after THA $[8,10,28]$, and they recommend hip surgery first. Ben-Gallim et al. published a study on the intervention of patients with LBP and hip OA. In this study, 25 patients were evaluated with pain and function scores before and after THA. All results showed a statistically significant improvement after THA. The authors concluded that THA relieved LBP and recommended hip surgery first [18]. However, since a small sample in this study may be considered a defect. In addition, another shortcoming of their study is that the patients they studied did not indicate whether there were pathological changes in the lumbar spine, which is very important for the interpretation of the results. Another study also found that patients with a history of lumbar spine fusion surgery to undergo THA had worse early outcomes and higher rates of complications and reoperation [27].

Combined with our follow-up results, for diagnosis and surgical treatment of patients with hip joint disease and lumbar degenerative disease, we suggest that:

We should focus on symptoms. In people with hips with pathological changes, the most common site of pain is in the buttocks, followed by thighs and groins [31]. However, lumbar degenerative disease can also lead to lower limb pain and dysfunction, which will lead to overlapping symptoms of the hip joint disease and lumbar degenerative disease, and needs further identification [32-34]. Detailed physical examination is helpful to the diagnosis of the disease. Patients with hip OA usually have inguinal pain, claudication or hip internal rotation limitation, which can induce lower limb pain during weight-bearing, hip internal rotation and external rotation, and lower limb rolling test [35]. Imaging examination can also provide further diagnostic information. Patients with hip discomfort can be examined by X-ray at the first visit, pathological changes of hip osteoarthritis include osteophyte hyperplasia at the edge of the femoral head and acetabular fossa, subchondral bone cyst formation and joint space stenosis [36] or early osteonecrosis through MRI examination [37,38]. At the same time, it is necessary to identify the lesions around the hip joint, such as acetabular dysplasia, glenoid lip tear, round ligament tear, synovitis, trochanteric bursitis, etc. If the cause of the current symptoms can be determined before the operation, then appropriate treatment can be carried out. However when there are pathological changes in the hip joint and spine, we recommend THA first. But patients also need to be told that performing surgery on one anatomical site can relieve the symptoms, but may also exacerbate symptoms in another. And the following situations need to be considered separately, serious lumbar degenerative diseases, such as cauda equina compression, lumbar spondylolisthesis leading to significant spinal canal stenosis. These patients need lumbar surgery first, but they also need to receive THA following the lumbar surgery, because the symptoms caused by hip joint diseases will not be relieved before THA. 
This retrospective study discusses the effect of THA on lumbar disease and analyzes for the first time the effect of unilateral and bilateral THA surgery on such patients. Meanwhile, this study used effective and reliable questionnaire for function and pain, with each patient as his/her own self-control. This study also has limitations. The most important limitation is that we did not analyze the imaging data of spine and pelvis in these patients after hip surgery, which may help us to further explore the pain relief mechanism. The next step of our study plan is to follow them up for a long time to see which patients need to undergo lumbar surgery following the THA. These limitations should be taken into consideration in interpreting our results and should be addressed in future studies.

\section{Conclusions}

The results of this study highlight the need to treat hip osteoarthritis first when patients have both hip disease (hip osteoarthritis, osteonecrosis of the femoral head, or both) and lumbar degenerative disease. After THA, LBP in some patients will be effectively relieved, thus avoiding further spinal surgery. Of course, for patients with cauda equina compression or severe lumbar lesions, more detailed and prudent surgical planning is needed.

\section{Abbreviations}

Total hip arthroplasty (THA), low back pain (LBP), Harris Hip score (HHS), Visual analysis scale (VAS), Japanese Orthopedic Association (JOA), lumbar disc herniation (LDH), degenerative lumbar spondylolisthesis (DLS), degenerative scoliosis (DS) lumbar spinal stenosis (LSS), computed tomography (CT), magnetic resonance imaging (MRI), recovery rate(RR), hip spine syndrome (HSS), osteoarthritis (OA)

\section{Declarations}

\section{Ethics approval and consent to participate}

All subjects received and accepted informed consent prior to participating in the study, which was approved by the Institutional Review Board of Xinqiao hospital.

\section{Consent to publish}

All the co-authors agreed to publish the article

\section{Availability of data and materials}

Not applicable

\section{Competing interests}


The authors declare there is no conflicts of interest and non-financial competing interests regarding the publication of this paper.

\section{Funding}

The design and collection of the study was supported by the National Key Research and Development Plan (grant numbers: 2016YFC1101900). The analysis, and interpretation of data was supported by Application of 3D printed individualized tibial and femoral osteotomy guide plate in accurate total knee replacement and application(2016YLC21) and writing the manuscript was supported by High bioactive 3D accurate printing of intraosseous plant materials and key technologies(2018C028).

\section{Authors' Contributions}

Dr. Wang conceived and designed the study. Dr. ran, Dr. Ke and Dr. Song completed the follow-up and collected scores. Dr. Lv and Dr. Li collected the patient's surgical information. Miss. Zhang and Miss. Zhou completed the statistical analysis. Dr. Ran wrote the paper. Dr. Wang edited the manuscript. All authors read and approved the manuscript. Tianfei Ran and Song Ke contributed equally to this work.

\section{Acknowledgements}

I would like to thank Professor Wang for his guidance on writing the article, as well as the National Key Research and Development Plan Fund for its sponsorship of the research.

\section{References}

1. F W (1999) Determinants of WOMAC function, pain and stiffness scores: evidence for the role of low back pain, symptom counts, fatigue and depression in osteoarthritis, rheumatoid arthritis and fibromyalgia. Rheumatology (Oxford, England) 38 (4):355-361.

doi:10.1093/rheumatology/38.4.355.

2. DJ B, BT N, MP TMS B. The Impact of Lumbar Spine Disease and Deformity on Total Hip Arthroplasty Outcomes. The Orthopedic clinics of North America. 2016;47(1):19-28. doi:10.1016/j.ocl.2015.08.005.

3. SB NL, PF G, KB L W (2019) Hip or spine surgery first?: a survey of treatment order for patients with concurrent degenerative hip and spinal disorders. The bone joint journal:37-44. doi:10.1302/0301620x.101b6.Bjj-2018-1073.R1.

4. BG D, GA B, PW G, Q SLLMDRA, PG N, AV P, CB M P. Complications in Patients Undergoing Spinal Fusion After THA. Clin Orthop Relat Res. 2018;476(2):412-7. doi:10.1007/s11999.0000000000000009.

5. M JSSOSKJN, G I TTSMTYE, S O MT, T I, G NOKKYA, M A, Y MHKMSYS, K OGK, K ITS, T Y T, K T. Difficulty of diagnosing the origin of lower leg pain in patients with both lumbar spinal stenosis and hip joint osteoarthritis. Spine. 2012;37(25):2089-93. doi:10.1097/BRS.0b013e31825d213d. 
6. H P, L vD (2019) Links between the Hip and the Lumbar Spine (Hip Spine Syndrome) as they Relate to Clinical Decision Making for Patients with Lumbopelvic Pain. PM \& R: the journal of injury, function, and rehabilitation:S64-S72. doi:10.1002/pmrj.12187.

7. PH H, DW YC, MS C, HN L, SW S U. Pain distribution and response to total hip arthroplasty: a prospective observational study in 113 patients with end-stage hip disease. Journal of orthopaedic science: official journal of the Japanese Orthopaedic Association. 2012;17(3):213-8. doi:10.1007/s00776-012-0204-1.

8. T E, S N, H B, P F, G G, O R (2017) Lumbar surgery prior to total hip arthroplasty is associated with worse patient-reported outcomes. The bone \& joint journal (6):759-765. doi:10.1302/0301620x.99b6.Bjj-2016-0577.R2.

9. M PS W, D P, J dB. Total joint arthroplasty and preoperative low back pain. J Arthroplast. 2014;29(5):867-71. doi:10.1016/j.arth.2013.10.001.

10. PC C, CJ D, CA WL, PD L, RJ F OK. Factors Associated With Early Improvement in Low Back Pain After Total Hip Arthroplasty: A Multi-Center Prospective Cohort Analyses. J Arthroplast. 2016;31(1):176-9. doi:10.1016/j.arth.2015.07.028.

11. AE JP, PF PAHGG S, RH R. Back pain and total hip arthroplasty: a prospective natural history study. Clin Orthop Relat Res. 2010;468(5):1325-30. doi:10.1007/s11999-010-1236-5.

12. TE B, JT BLFS M. Thigh pain after cementless total hip arthroplasty: evaluation and management. J Am Acad Orthop Surg. 2002;10(6):385-92. doi:10.5435/00124635-200211000-00002.

13. GM R, BA M, DS G, CP D. Evaluation of pain in patients with apparently solidly fixed total hip arthroplasty components. J Am Acad Orthop Surg. 2002;10(2):86-94. doi:10.5435/00124635200203000-00004.

14. WH H. Traumatic arthritis of the hip after dislocation and acetabular fractures: treatment by mold arthroplasty. An end-result study using a new method of result evaluation. The Journal of bone joint surgery American volume. 1969;51(4):737-55.

15. BP MYLYBZ, XT XNC, SD W, YJ C, XJ W C. Simplified Chinese Version of the Japanese Orthopaedic Association Back Pain Evaluation Questionnaire: Agreement, Responsiveness, and Minimal Important Change for Patients With Chronic Low Back Pain. Spine. 2018;43(20):1438-45. doi:10.1097/brs.0000000000002692.

16. T F, S O, M I, R Y, T M, T Y, T M, E W, T O. Validity of the Japanese Orthopaedic Association scoring system based on patient-reported improvement after posterior lumbar interbody fusion. The spine journal: official journal of the North American Spine Society. 2016;16(6):728-36. doi:10.1016/j.spinee.2016.01.181.

17. EC JS H. Graphic representation of pain. Pain. 1976;2(2):175-84. doi:10.1016/0304-3959(76)901147.

18. P B-G, T B-G NR, A H, J H, S D, Y F. Hip-spine syndrome: the effect of total hip replacement surgery on low back pain in severe osteoarthritis of the hip. Spine. 2007;32(19):2099-102. doi:10.1097/BRS.0b013e318145a3c5. 
19. K O, T HY T, Y M, R K, H S. Comparison of patient satisfaction after unilateral or simultaneous bilateral total hip arthroplasty through a direct anterior approach: Evaluation using the Japanese Orthopaedic Association Hip Disease Evaluation Questionnaire. Journal of orthopaedic science: official journal of the Japanese Orthopaedic Association. 2016;21(3):332-5. doi:10.1016/j.jos.2016.01.008.

20. O H DE, M S, N B, V D, A C. Lumbar spinal stenosis: assessment of cauda equina involvement by electrophysiological recordings. Journal of neurology. 2007;254(6):741-50. doi:10.1007/s00415006-0427-1.

21. A P, C GSDBVMPB, C L, B M M. Erratum to: Spinopelvic parameter changes and low back pain improvement due to femoral neck anteversion in patients with severe unilateral primary hip osteoarthritis undergoing total hip replacement. European spine journal: official publication of the European Spine Society, the European Spinal Deformity Society, and the European Section of the Cervical. Spine Research Society. 2018;27(1):135. doi:10.1007/s00586-017-5092-9.

22. LA KEBESB N, U K. Effects of total hip arthroplasty on spinal sagittal alignment and static balance: a prospective study on 28 patients. European spine journal: official publication of the European Spine Society, the European Spinal Deformity Society, and the European Section of the Cervical. Spine Research Society. 2016;25(11):3615-21. doi:10.1007/s00586-016-4696-9.

23. W W, H W, M W, Y Z, Y Q, W W. The effect of total hip arthroplasty on sagittal spinal-pelvic-leg alignment and low back pain in patients with severe hip osteoarthritis. European spine journal: official publication of the European Spine Society, the European Spinal Deformity Society, and the European Section of the Cervical. Spine Research Society. 2016;25(11):3608-14. doi:10.1007/s00586-016-4444-1.

24. CM O IM. Hip-spine syndrome. Spine. 1983;8(3):316-21. doi:10.1097/00007632-198304000-00014.

25. CJ D, KA M, BJ M, AJ Y, JD K. Hip-spine syndrome. J Am Acad Orthop Surg. 2012;20(7):434-42. doi:10.5435/jaaos-20-07-434.

26. LM D, PL EMDBMB, JY Z, JC M, JM T V, R S, R L, V L, T P, AJ B. Radiological severity of hip osteoarthritis in patients with adult spinal deformity: the effect on spinopelvic and lower extremity compensatory mechanisms. European spine journal: official publication of the European Spine Society, the European Spinal Deformity Society, and the European Section of the Cervical. Spine Research Society. 2018;27(9):2294-302. doi:10.1007/s00586-018-5509-0.

27. JJ B, DC S, TP V, EN H. Early Outcomes of Primary Total Hip Arthroplasty After Prior Lumbar Spinal Fusion. J Arthroplast. 2017;32(2):470-4. doi:10.1016/j.arth.2016.07.019.

28. A P, C GSDBVMPB, C L, B M M. Spinopelvic parameter changes and low back pain improvement due to femoral neck anteversion in patients with severe unilateral primary hip osteoarthritis undergoing total hip replacement. European spine journal: official publication of the European Spine Society, the European Spinal Deformity Society, and the European Section of the Cervical. Spine Research Society. 2018;27(1):125-34. doi:10.1007/s00586-017-5033-7. 
29. J C (1961) Arthroplasty of the hip. A new operation. Lancet (London, England) 1 (7187):1129-1132. doi:10.1016/s0140-6736(61)92063-3.

30. TH M, EA S, CS R, PD W. A fifteen-year follow-up study of one hundred Charnley low-friction arthroplasties. The Orthopedic clinics of North America. 1988;19(3):467-76.

31. Borowsky C, Fagen G. Sources of sacroiliac region pain: insights gained from a study comparing standard intra-articular injection with a technique combining intra- and peri-articular injection. Arch Phys Med Rehabil. 2008;89(11):2048-56. doi:10.1016/j.apmr.2008.06.006.

32. Li W-s, Gang L, Chen Z-q, Wood KB. Sagittal plane analysis of the spine and pelvis in adult idiopathic scoliosis. Chin Med J. 2010;123(21):2978-82.

33. Lesher JM, Dreyfuss $P$, Hager N, Kaplan M, Furman M. Hip joint pain referral patterns: a descriptive study. Pain Med. 2008;9(1):22-5.

34. Kahn C. Use of triamcinolone hexacetonide for the treatment of osteoarthritis of the hip: comment on the letter by Margules. Arthr Rhuem. 2002;46(9):2546-7.

35. Brown MD, Gomez-Marin O, Brookfield KF, Li PS. (2004) Differential diagnosis of hip disease versus spine disease. Clinical Orthopaedics and Related Research (1976-2007) 419:280-284.

36. NE L (2007) Clinical practice. Osteoarthritis of the hip. The New England journal of medicine 357 (14):1413-1421. doi:10.1056/NEJMcp071112.

37. K T, M TSHH T, N S. Which Classification System Is Most Useful for Classifying Osteonecrosis of the Femoral Head? Clin Orthop Relat Res. 2018;476(6):1240-9.

doi:10.1007/s11999.0000000000000245.

38. T Y SI, G M, Y N, T M, Y I. MRI evaluation of collapsed femoral heads in patients 60 years old or older: Differentiation of subchondral insufficiency fracture from osteonecrosis of the femoral head. AJR American journal of roentgenology. 2010;195(1):W63-8. doi:10.2214/ajr.09.3271.

\section{Figures}



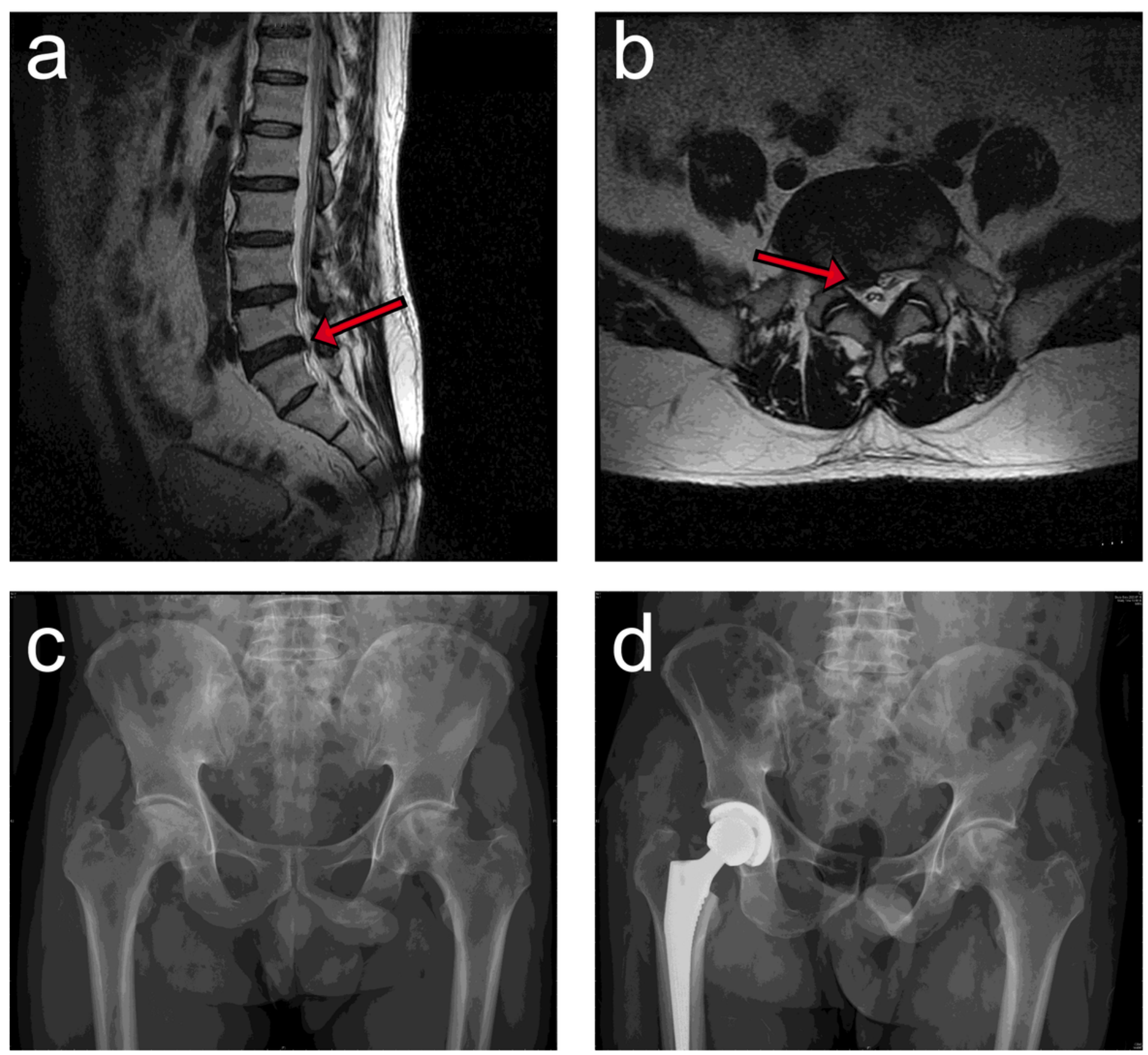

Sagittal image of lumbar spine on T2WI (a), Axial image of lumbar spine on T2WI (b) Preoperative X-ray of the pelvis (c), Postoperative X-ray of the pelvis (d)

\section{Figure 1}

A 49-year-old man with lumbar degenerative disease and bilateral osteonecrosis of the femoral head. He had lumbar disc herniation (L5-S1) and bilateral osteonecrosis of the femoral head, because the pain on the left was mild and the pain on the right was obvious, so he only received total hip arthroplasty on the right. 


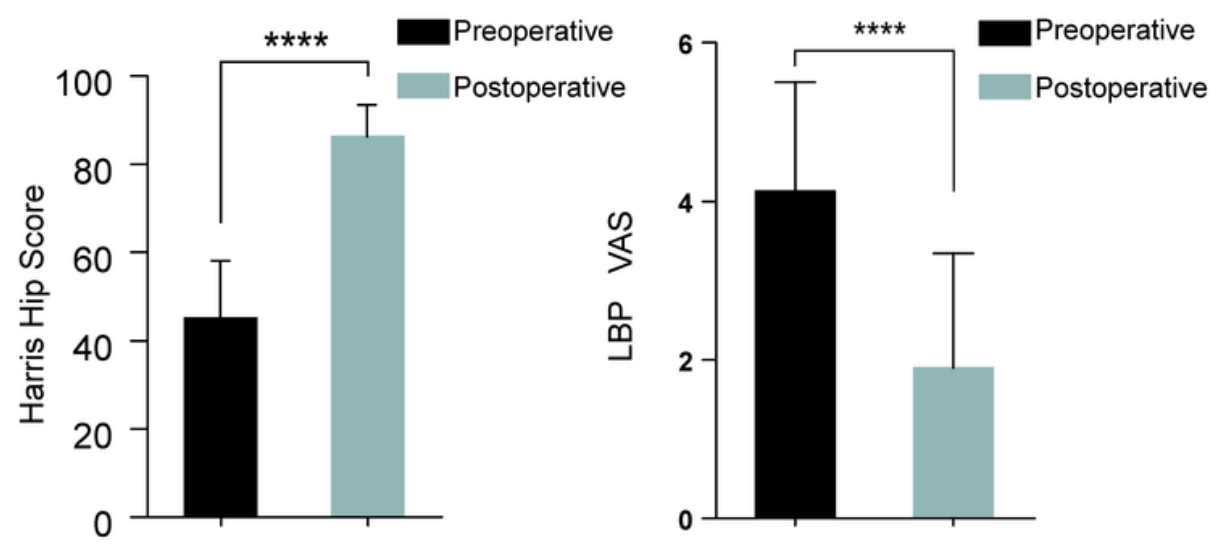

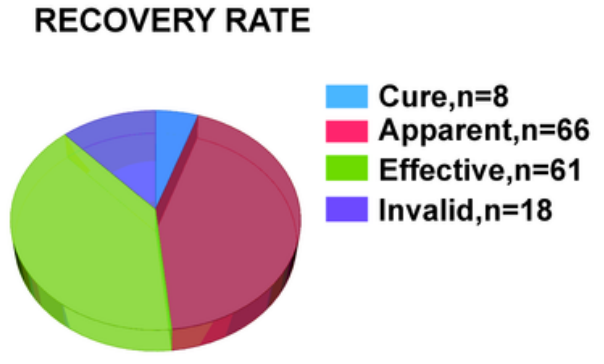

Recovery rate:

Cure $(R R=100 \%) 、$ Apparent $(60 \% \leqq R R<99 \%)$

Effective $(25 \% \leq R R<60 \%)$ 、 Invalid $(R R<25 \%)$

Figure 2

Data on Low back pain (LBP) VAS, recovery rate (RR) and hip function pre-and-post hip arthroplasty. Based on $t$ tests, comparing the data of pre-hip operation-with that of post-hip operation there was a significant improvement $(P<0.0001)$ in the Harris Hip scores for pain relief and function recovery of hip, and there was a significant decrease $(P<0.0001)$ in the LBP VAS. Based on the calculation of RR in JOA scoring system, that 8 patients were cure, 66 were apparent, 61 were effective, and 10 were invalid. All values are statistically calculated as Mean $\pm S D$. ${ }^{\star * \star \star} P<0.0001$
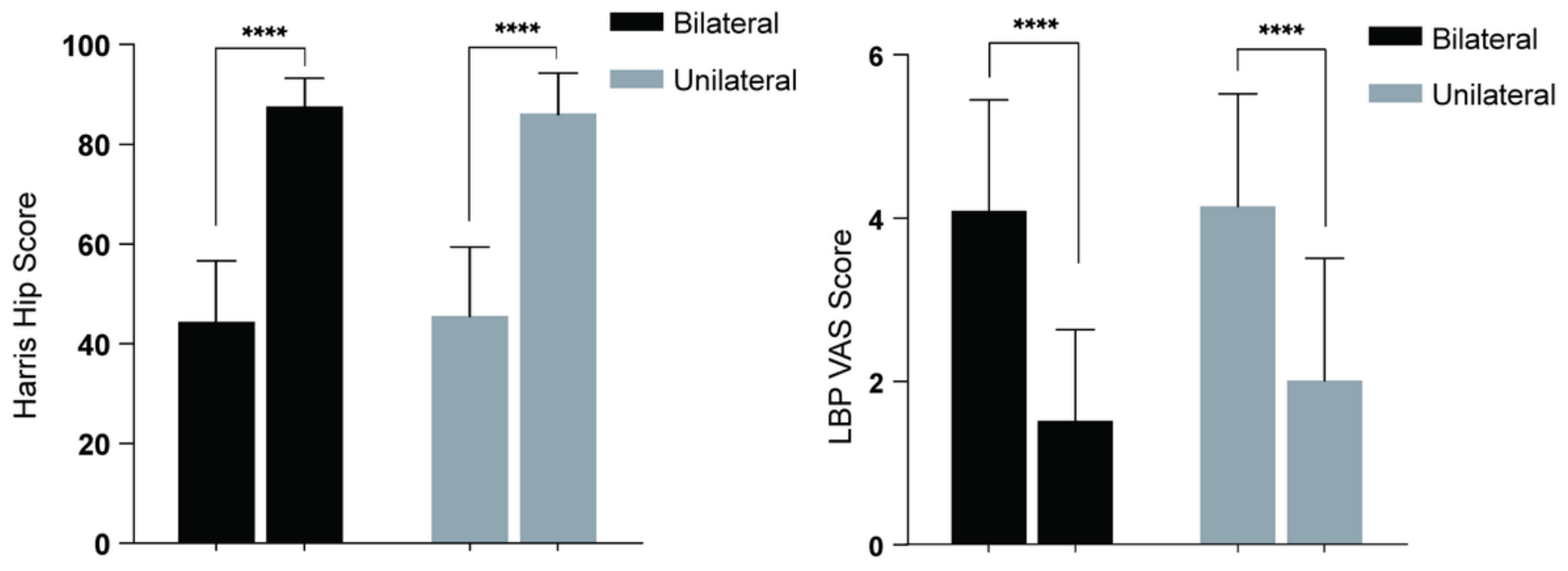

Figure 3

LBP VAS and Harris Hip score in bilateral and unilateral pre- and -post THA. Based on the t-tests, the LBP VAS and Harris scores for pain relief and function recovery of hip in patients with bilateral and unilateral THA were significantly improved $(p<0.0001)$ after operation. All values are statistically calculated as Mean \pm SD. 